OPEN ACCESS

Edited by:

Mikhail Lebedev,

Duke University, United States

Reviewed by:

Pere Berbel,

Universidad Miguel Hernández de

Elche, Spain

Sergio E. Lew,

Universidad de Buenos Aires,

Argentina

Hanna Bronisława Cygan Institute of Physiology and Pathology

of Hearing (IFPS), Poland

*Correspondence:

André Fujita

fujita@ime.usp.br

Received: 10 September 2018 Accepted: 27 December 2018

Published: 15 January 2019

Citation:

Ramos TC, Balardin JB, Sato JR and Fujita A (2019) Abnormal Cortico-Cerebellar Functional

Connectivity in Autism Spectrum Disorder. Front. Syst. Neurosci. 12:74. doi: 10.3389/fnsys.2018.00074

\section{Abnormal Cortico-Cerebellar Functional Connectivity in Autism Spectrum Disorder}

\author{
Taiane Coelho Ramos ${ }^{1}$, Joana Bisol Balardin ${ }^{2}$, João Ricardo Sato ${ }^{3}$ and André Fujita ${ }^{1 *}$ \\ ${ }^{1}$ Department of Computer Science, Institute of Mathematics and Statistics, University of São Paulo, São Paulo, Brazil, ${ }^{2}$ Brain \\ Institute, Hospital Israelita Albert Einstein, São Paulo, Brazil, ${ }^{3}$ Center of Mathematics, Computation, and Cognition, \\ Universidade Federal do ABC, Santo André, Brazil
}

The cerebral cortex and the cerebellum are spatially remote areas that are connected by complex circuits that link both primary and associative areas. Previous studies have revealed abnormalities in autism spectrum disorder (ASD); however, it is not clear whether cortico-cerebellar connectivity is differentially manifested in the disorder. To explore this issue, we investigated differences in intrinsic cortico-cerebellar functional connectivity between individuals with typical development (TD) and those with ASD. To this end, we used functional magnetic resonance imaging (fMRI) of 708 subjects under a resting state protocol provided by the ABIDE I Consortium. We found that people with ASD had diminished functional connectivity between the cerebellum and the following cortical regions: (i) right fusiform gyrus, (ii) right postcentral gyrus, (iii) right superior temporal gyrus, (iv) right middle temporal gyrus, and (v) left middle temporal gyrus. All of these regions are involved in many cognitive systems that contribute to commonly affected functions in ASD. For right fusiform gyrus, right superior temporal gyrus, and left middle temporal gyrus, we reproduced the results in an independent cohort composed of 585 subjects of the ABIDE II Consortium. Our results points toward a consistent atypical cortico-cerebellar connectivity in ASD.

Keywords: cortico-cerebellar connectivity, autism spectrum disorders, ASD, resting-state fMRI, cerebellum, functional connectivity, underconnectivity, ABIDE

\section{INTRODUCTION}

Autism spectrum disorders (ASD) are mainly characterized by repetitive behavior and social impairment, including differentiated sensitivity to sound and touch and difficulty in recognizing non-verbal language and facial expressions (American Psychiatric Association, 2013). These symptoms may affect a child's cognitive development, which may prevent self-sufficiency in adulthood. One out of 68 children in the U.S. (Christensen, 2016) and 1\% of the population worldwide (Elsabbagh et al., 2012) are estimated to have ASD. Despite this high incidence, the pathophysiology of ASD is still unclear. Therefore, additional studies are needed to better understand the underlying mechanisms and develop proper treatments for ASD.

Cerebellar abnormalities have been implicated in ASD (Fatemi et al., 2012), as suggested by studies correlating ASD to a reduced number and size of Purkinje cells (Fatemi et al., 2002; Bauman and Kemper, 2005) and cerebellar vermis hypoplasia (Courchesne et al., 1988; Hashimoto et al., 1995; Webb et al., 2009). Moreover, cerebellar lesions in premature children may result in symptoms similar to ASD (Limperopoulos et al., 2014). 
Although the cerebellum was originally considered a motor structure, (Schmahmann, 2010; Noroozian, 2014; Baumann et al., 2015; Hoche et al., 2016) identified its role in cognitive, social, and emotional abilities, in special by observations of the cerebellar cognitive affective syndrome (Schmahmann, 2004). Its involvement in these functions may be explained by the vast white matter pathways connecting the cerebellum to functionally heterogeneous cortical regions (Glickstein, 1992; Ramnani, 2006). Functional cortical-cerebellar connectivity studies suggest a correlation between the cerebellar activity and several cortical regions, particularly correlation between the cerebellar frontal lobe and temporal, auditory, superior temporal, somatosensory, motor, and premotor regions and between the cerebellar posterior lobe and posterior parietal regions and the prefrontal cortex (O'Reilly et al., 2010; Buckner et al., 2011). In addition, meta-studies report cerebellar activity related to higher cognitive domains, including language, verbal working memory, and emotional processing (Stoodley and Schmahmann, 2009; Buckner, 2013; Keren-Happuch et al., 2014).

Currently, ASD is believed to be a disorder related more to differential brain connectivity than to the activity in a specific brain region (Müller et al., 2011; Maximo et al., 2014). Thus, it is natural to ask whether cortico-cerebellar connections are differentiated in ASD compared to typical development (TD). Attempts to answer this question are usually based on diffusion imaging studies, most of which show decreased Fractional Anisotropy (FA) suggesting a weaker structural connectivity in participants with ASD (Catani et al., 2008; Brito et al., 2009; Hanaie et al., 2013) (for a review, see Crippa et al., 2016). Task-driven fMRI studies reported decreased functional corticocerebellar connectivity during finger tapping task (Mostofsky et al., 2009) and verb generation task (Verly et al., 2014) in children with ASD.

A potential technique for studying neural connectivity is resting-state fMRI (rs-fMRI). rs-fMRI measures fluctuations in the blood oxygen level-dependent (BOLD) signal when a subject is not performing any specific task (Biswal et al., 1995). The brain areas and their respective correlations between the BOLD signals form the functional network (Fox and Raichle, 2007), which has been shown to be a good approximation of structural connections (Smith et al., 2009). However, few rsfMRI studies focus specifically on cortico-cerebellar connectivity alterations in ASD. To the best of our knowledge, the study by Khan et al. (2015) is the only one that aimed at assessing functional cortico-cerebellar connectivity using rs-fMRI. The authors found a general cortico-cerebellar overconnectivity in children and adolescents with ASD, in special in sensori-motor networks, accompanied by underconnectivity in supramodal networks. They relate these results with previous findings of early overgrowth of the white matter, possibly leading to poorly assembled networks.

Considering the evidence, the current study aimed to identify brain regions with differential functional connectivity with the cerebellum in ASD, using rs-fMRI in a public large discovery sample (ABIDE I dataset) and to validate the findings in a validation sample (ABIDE II dataset). To the best of our knowledge, this is the first work to assess functional cortico-cerebellar connectivity that reproduced results on an independent dataset.

\section{MATERIALS AND METHODS}

To test our hypothesis of differential cortico-cerebellar functional connectivity between subjects with TD and those with ASD, we downloaded a large fMRI dataset from the ABIDE I Consortium and confirmed the results using an independent dataset (ABIDE II).

\subsection{Functional MRI Data}

We downloaded two large resting-state fMRI datasets, namely, ABIDE I and ABIDE II. ABIDE I is composed of 573 individuals with TD and 539 individuals with ASD (totaling 1112 subjects). ABIDE II is composed of 593 individuals with TD and 521 individuals with ASD (totaling 1114 subjects). After preprocessing (described in section 2.2.), the ABIDE I dataset was composed of 432 subjects with TD (348 males, mean age \pm standard deviation of $18.21 \pm$ 8.04) and 276 individuals with ASD (241 males, $18.42 \pm$ 8.38). The ABIDE II dataset was composed of 316 subjects with TD (208 males, $12.80 \pm 5.61)$ and 269 individuals with ASD (234 males, $13.93 \pm 7.07)$. Both are available on the ABIDE Consortium website (http://fcon_1000.projects. nitrc.org/indi/abide/). According to the ABIDE repository, the acquisition methods and protocols were approved by the corresponding local Institutional Review Boards (i.e., the review boards and their regulations at the California Institute of Technology, Carnegie Mellon University, ETH Zürick, Georgetown University, Indiana University, Kennedy Krieger Institute, University of Leuven, Ludwig Maximilians University Munich, New York University, Oregon Health and Science University, Institute of Living at Hartford Hospital, University of Pittsburgh, Social Brain Lab, San Diego State University, Stanford University, Trinity Center for Health Sciences, University of California Davis, University of California Los Angeles, University of Michigan, University of Utah School of Medicine, Yale School of Medicine) and were performed in accordance with Health Insurance Portability and Accountability Act (HIPAA) guidelines and the 1,000 Functional Connectomes Project/International Data-sharing Initiative (http://fcon_1000. projects.nitrc.org/) protocols. Written informed consent was obtained from all the participants. All data distributed via the ABIDE website were fully anonymized in compliance with the HIPAA privacy rules, and no protected health information was included. The imaging protocols are considered to be equivalent across different institutes. Further details about this dataset can be obtained from the ABIDE consortium website.

\subsection{Image Preprocessing}

We preprocessed the imaging data using the Athena pipeline (www.nitrc.org/plugins/mwiki/index.php/neurobureau:Athena Pipeline). The pipeline focused on providing systematic processing of fMRI data, including the following main steps: exclusion of the first four scans; slice timing correction; 
deoblique dataset; correction for head movements; masking the volumes to exclude non-brain regions; co- registration of the mean image to the respective anatomic image of the subject; spatial normalization to $\mathrm{MNI}$ space $(4 \times 4 \times 4 \mathrm{~mm}$ resolution); extraction of BOLD (Ogawa et al., 1990) time series from white matter (WM) and cerebrospinal-fluid (CSF); removing the effects of WM, CSF, motion, and trend using multiple linear regression; temporal band-pass filter $(0.009<f<0.08 \mathrm{~Hz})$; and spatially smoothing the filtered data using a Gaussian filter (FWHM $=6 \mathrm{~mm}$ ). All these steps were performed by using the following software: Analysis of Functional NeuroImages (AFNI) (http://afni.nimh.nih.gov/afni) and the fMRIB Software Library (FSL) (http://fsl.fMRIb.ox.ac.uk/fsl/fslwiki/). To define the 116 ROIs considered in this study, we used the Automated Anatomical Labeling (AAL) atlas (Tzourio-Mazoyer et al., 2002). We identified 25 ROIs comprising the ventricles by using the Montreal Neurological Institute (MNI) atlas, and we removed them. The head coil coverage available in the scanners may vary depending on the model, which may lead to a lack of reading of part of the cerebellum for some subjects. Thus, to avoid artifacts, we adopted a masking procedure to constrain the statistics only to ROIs with a mean voxels sampling of $80 \%$ across participants. An image was created from the overlay plot to represent the percentage of valid voxels in each cerebellar ROI (see Figure S1). We found that ROIs from I-VI lobes and the vermis region matched this criterion. Thus, we considered 79 ROIs ( 65 cortical and 14 cerebellar) for further analysis. Subjects' head movements during MRI scanning could lead to spurious correlations between ROIs; thus, we carried out the "scrubbing" procedure (Power et al., 2012) to remove frames affected by head movement. We removed frames that presented both framewise displacement (FD) greater than $0.5 \mathrm{~mm}$ and DVARS greater than $0.5 \% \triangle B O L D$ (Power et al., 2012). Preceding and following frames that did not meet these criteria were not removed. Subjects that had more than $5 \%$ of the total number of scans removed by scrubbing were excluded from the analysis. Head motion across the dataset is measured by the FD, which presents a mean of $0.137 \mathrm{~mm}$ and standard deviation of $0.102 \mathrm{~mm}$ on the study dataset (ABIDE I) and a mean of $0.169 \mathrm{~mm}$ with standard deviation of $0.132 \mathrm{~mm}$ on the independent dataset (ABIDE II). A total of 93 subjects (53 TD and 40 ASD) in the study dataset (ABIDE I) and 45 subjects (24 TD and 21 ASD) in the independent dataset (ABIDE II) had scans removed by the scrubbing process.

We considered both autism and Asperger syndrome as parts of the ASD as suggested by the Diagnostic and Statistical Manual of Mental Disorders 5th edition (DSM-5) (American Psychiatric Association, 2013).

\subsection{Cortico-Cerebellar Functional Connectivity Analysis}

To summarize, reduce the number of variables, and enhance the statistical power, for each subject in the sample, we applied the principal component analysis (PCA) on the 14 cerebellar ROI time series. Then, for each subject, we selected the principal components (PCs) that explained at least 95\% of the data variance. Thus, each subject had a different number of PCs used for the analyses (average of 9.25 PCs with standard deviation of 1.19).

Next, to identify the cortical regions that are functionally associated with the cerebellum, for each subject, we carried out a linear regression with the cortical ROI time series as response variables and the PCs of the cerebellum obtained by the PCA for the subject as predictor variables. The adjusted $R^{2}$ (coefficient of determination) was considered as a measure of functional connectivity between the cortical ROI and the cerebellum.

To test if the functional connectivity was different between the TD and ASD groups for each cortical ROI, we performed a linear regression using the measure of functional connectivity (the adjusted $R^{2}$ obtained for each subject in the previous step) as the response variable and the diagnostic group (TD or ASD) as the predictor variable. To reduce age, gender, and site effects, we included them as covariates in this linear model. All p-values were corrected for multiple tests by using the False Discovery Rate (FDR) (Benjamini and Hochberg, 1995) approach (we considered all 65 tests, one for each cortical ROI). The corrected $p$-value threshold considered to be statistically significant was set at $5 \%$.

\section{RESULTS}

First, we carried out the procedures described in the sections 2.2. and 2.3. using the ABIDE I dataset. In summary, for each subject, we carried out a linear regression between the fMRI time series of each cortical ROI and the cerebellum. To represent the neural activity of the cerebellum, we used the cerebellar PCs representing $95 \%$ of the variance of the time series in this region. We estimated the $R^{2}$ value of this regression as a measure of connectivity between each cortical ROI and the cerebellum as a whole. Then, we carried out a second linear regression between the $R^{2}$ values obtained in the previous linear regression and the group (TD or ASD), by including age, gender, and site as covariates. We identified five cortical ROIs (Figure 1) that are differentially associated with the cerebellum between the typical development (TD) and autism spectrum disorder (ASD) groups, namely, the right fusiform gyrus $[\beta=-0.042, t$-value $=-3.689$, $p=0.005-t$-test (GLM) $]$, the right postcentral gyrus $[\beta=$ $-0.038, t$-value $=-3.131, p=0.027-\mathrm{t}$-test $(\mathrm{GLM})]$, the right superior temporal gyrus $[\beta=-0.034, t$-value $=-3.082$, $p=0.027-t$-test $(\mathrm{GLM})]$, the right middle temporal gyrus $[\beta=-0.039, t$-value $=-3.783, p<0.001-t$-test $(\mathrm{GLM})]$, and the left middle temporal gyrus $[\beta=-0.056, t$-value $=$ $-5.481, p=0.005-t$-test (GLM)]. All p-values were corrected for FDR. Figure 2 presents the barplots for the cortico-cerebellar functional connectivity measurements (adjusted $R^{2}$ ) for the TD and ASD groups for each of the five cortical ROIs. It is important to mention that for all of the identified cortical regions, the cortico-cerebellar functional connectivity in the ASD group was statistically lower than that in the TD group.

Then, to check the reproducibility of the findings, we tested these five differential cortico-cerebellar connectivity areas on an independent dataset composed of 585 subjects collected from 11 sites available in the ABIDE II consortium. The criteria for selecting the participants and the preprocessing procedure adopted for the independent dataset were the same 


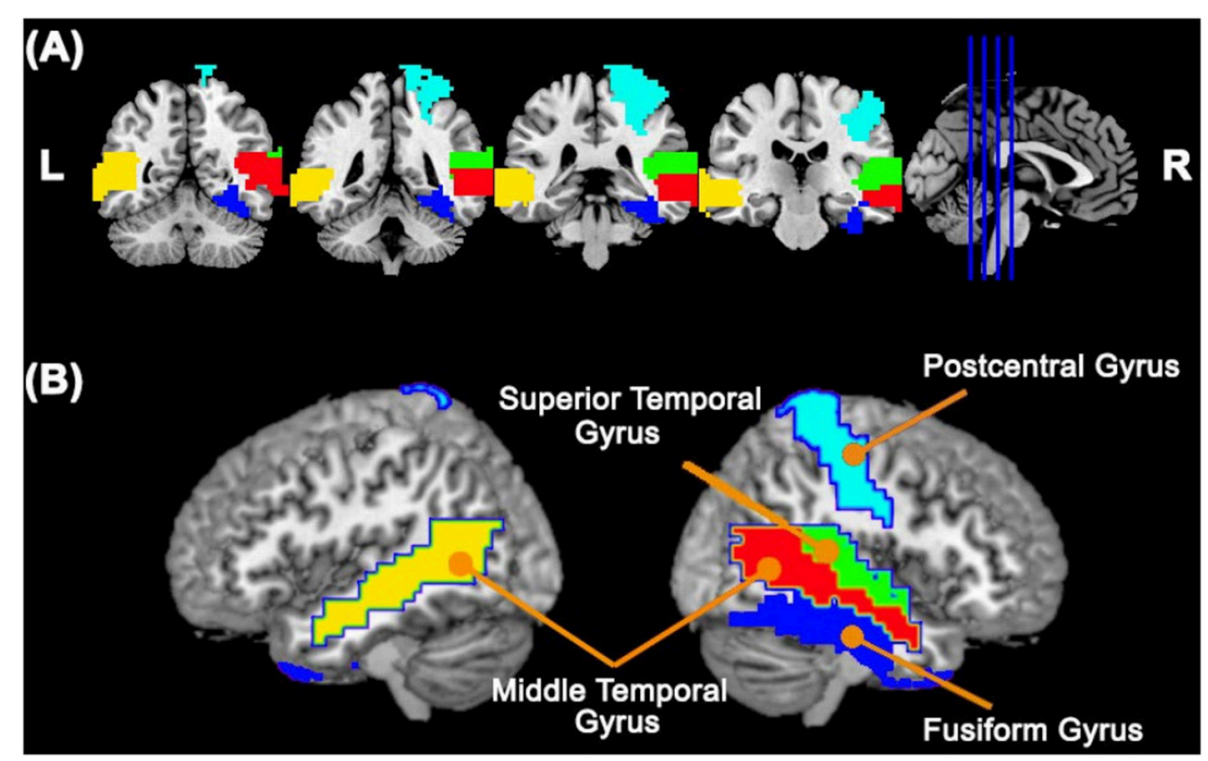

FIGURE 1 | Cortical ROls with a differential association with the cerebellum between TD and ASD groups obtained by analyzing the ABIDE I dataset. Panels (A,B) represent coronal slices and two lateral views of the brain, respectively. The colors represent the cortical ROls that differentially associated with the cerebellum between TD and ASD groups, namely, the right fusiform gyrus, right postcentral gyrus, right superior temporal gyrus, right middle temporal gyrus, and left middle temporal gyrus.

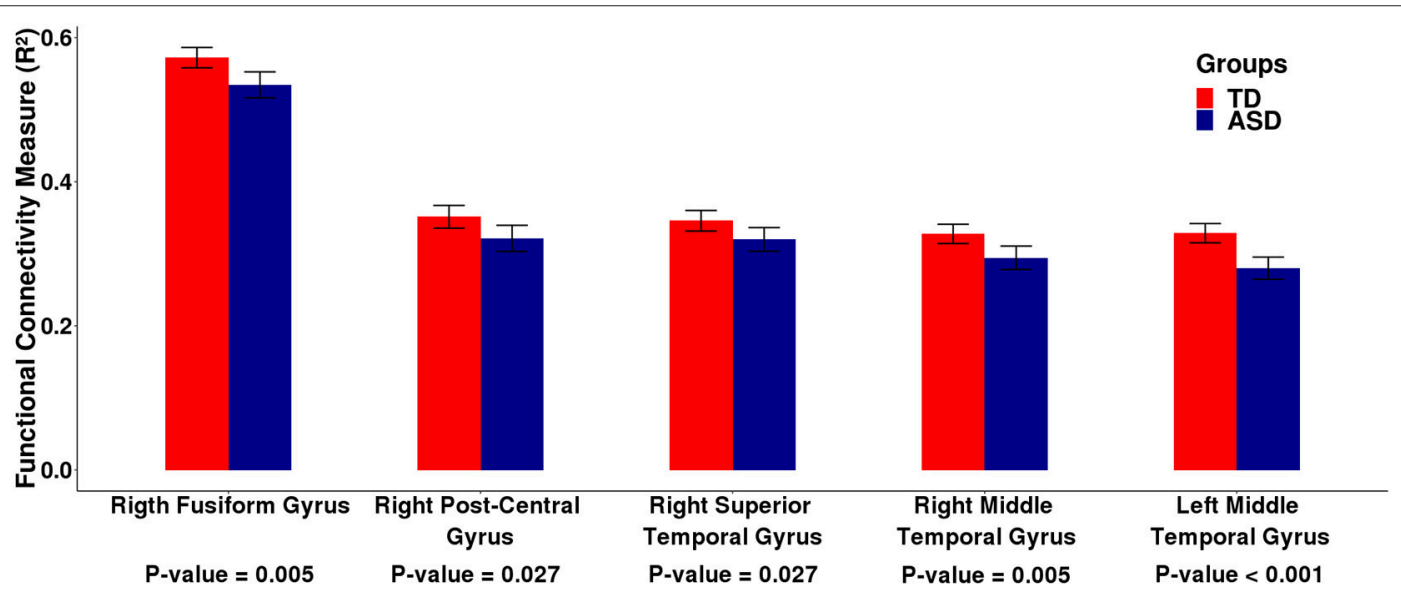

FIGURE 2 | Barplots of the functional connectivity measure $\left(R^{2}\right)$ between the cortical ROI and the cerebellum obtained by analyzing the ABIDE I dataset. Each pair of bars represent the mean functional connectivity measurements between the cortical ROI and the cerebellum for the TD and ASD groups, respectively. The error bars represent 95\% confidence interval. $P$-values were obtained by linear regression with the measure of functional connectivity $\left(R^{2}\right)$ as the response variable and the diagnostic group (TD or ASD) as the predictor variable with age, gender, and site as covariates. All $p$-values were corrected for multiple tests by FDR. Note that the mean functional connectivity measurement $\left(R^{2}\right)$ is significantly lower in the ASD group compared to the TD group, suggesting decreased functional cortico-cerebellar connectivity in the ASD group.

as described for the ABIDE I dataset in the section 2.2. In this validation analysis, three out of five cortical ROIs, namely, the right fusiform gyrus $[\beta=-0.028, t$-value $=-2.193$, $p=0.047-t$-test $(\mathrm{GLM})]$, the right superior temporal gyrus $[\beta=-0.044, t$-value $=-3.495, p=0.002-t$-test $(\mathrm{GLM})]$, and the left middle temporal gyrus $[\beta=-0.033, t$-value $=-2.583, p=0.025-t$-test (GLM) $]$, confirmed the reduced functional connectivity in the ASD group ( $p$-values corrected for multiple tests by FDR). The barplots for the functional connectivity measures (adjusted $R^{2}$ ) of these three cortical regions are shown in Figure 3. It is important to mention that the cortico-cerebellar functional connectivity in the ASD group was statistically lower than that in the TD group, as observed in the previous dataset. For some brief results on difference in intracortical connectivity between groups, see Figures S2, S3. 


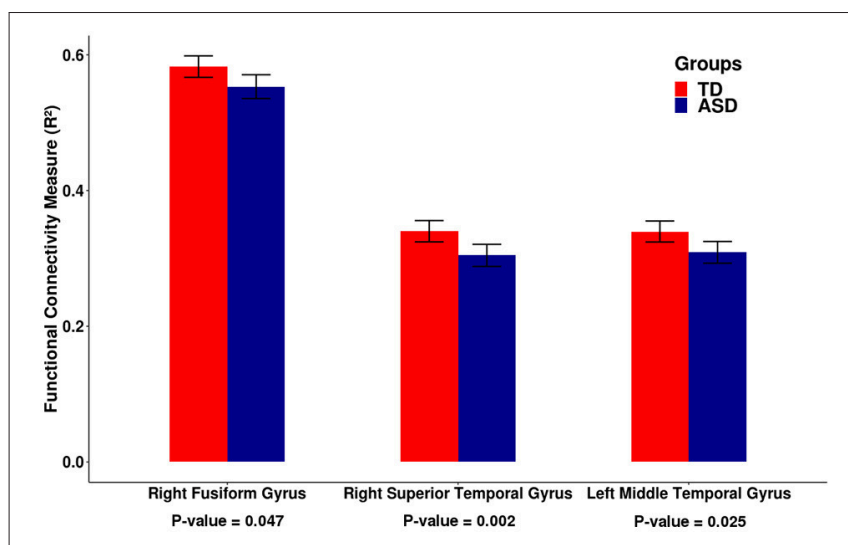

FIGURE 3 | Barplots of the functional connectivity measure $\left(R^{2}\right)$ between the cortical ROI and the cerebellum for an independent dataset (ABIDE II). Each pair of bars represent the mean functional connectivity measurements between the cortical ROI and the cerebellum for the TD and ASD groups, respectively. The error bars represent 95\% confidence interval. $P$-values were obtained by linear regression with the measure of functional connectivity $\left(R^{2}\right)$ as the response variable and the diagnostic group (TD or ASD) as the predictor variable with age, gender, and site as covariates. All $p$-values were corrected for multiple tests by FDR. Note that the median functional connectivity measurement $\left(R^{2}\right)$ is significantly lower in the ASD group compared to the TD group, suggesting decreased functional cortico-cerebellar connectivity in the ASD group. These data confirm the findings that were obtained by analyzing the ABIDE I dataset.

\section{DISCUSSION}

In this study, we investigated the differences in the intrinsic cortico-cerebellar functional connectivity between individuals with TD and those with ASD. We found that people with ASD had diminished functional connectivity between the cerebellum and several cortical regions, including the right postcentral, middle temporal, superior temporal, and fusiform gyri and the left middle temporal gyrus. For the right fusiform gyrus, the right superior temporal gyrus, and the left middle temporal gyrus, the validity of the results was confirmed in a large independent sample (ABIDE II).

Our results are in line with previous studies based on diffusion tensor imaging (DTI) suggesting alterations in structural connectivity in ASD. These studies show reduced values of fractional anisotropy (FA) in the white matter of individuals with ASD (Shukla et al., 2010; Libero et al., 2016) and abnormal development of such white matter pathways in young children on the spectrum (Ben Bashat et al., 2007; Wolff et al., 2012). In addition, there are studies showing specifically reduced FA in the temporal lobe (Barnea-Goraly et al., 2004; Lee et al., 2007), in agreement with our results of reduced functional connectivity in the right middle and superior temporal gyri and left middle temporal gyrus.

Abnormal functional connectivity in the cerebellum has been reported in ASD, albeit with mixed results. For example, cortico-cerebellar functional overconnectivity in the sensorimotor regions and underconnectivity in the supramodal regions were previously described in children and adolescents with ASD (Khan et al., 2015). In adolescents with ASD, reduced connectivity was also shown in the right crus I and several contralateral cerebral regions, including the superior frontal gyrus, middle frontal gyrus, thalamus, and anterior cingulate gyrus, as well as in the precentral gyrus (Verly et al., 2014). In our replication study, a prominent pattern of reduced connectivity in ASD was observed in the lateral temporal cortex. This variation among the results in the different studies may reflect differential alterations in connectivity related to patient characteristics (i.e., age, disease duration, and symptom severity) or image analysis methods, as previously discussed in the autism literature (Ecker et al., 2015). Although the nature and direction of functional connectivity differences in ASD remain inconclusive, our results add to the evidence indicating decreased cortico-cerebellar functional connectivity in patients.

The specific cortical regions that exhibited ASD-related altered functional connectivity with the cerebellum have also been linked to symptoms and traits of autism. For example, differences in frontocerebellar circuits have been associated with motor impairments and stereotyped repetitive behaviors (Floris et al., 2016), and the lateral temporal cortex and fusiform gyrus appear to mediate social processing deficits (for a review, see Just et al., 2012). Altogether, these findings suggest that differences in cortico-cerebellar functional connectivity in organized somatomotor and associative networks may contribute to the clinical manifestations in ASD, although further studies are needed to establish a direct association between our findings and autistic symptoms.

The neurobiological mechanisms underlying the diminished cortico-cerebellar functional connectivity in ASD observed in our study remain speculative. The communication of the cerebellum with the cerebral cortex has been associated with a closedloop system in which the cerebellum returns projections to the cerebral cortex via the thalamus (for a review, see Ramnani, 2006). In this context, cortico-cerebellar connectivity differences could emerge from developmental alterations in both thalamocortical and/or extrinsic cortico-cortical projections. In fact, prior ASD studies using diffusion tensor MRI have detected differences in microstructural integrity of tracts connecting thalamus with motor and somatosensory cortices (Nair et al., 2013), and also of the corpus callosum (Alexander et al., 2007). It is important to note, however, that microstructural alterations have also been reported in cerebellar tracts (e.g., intracerebellar fibers and right superior cerebellar output peduncle) (Catani et al., 2008). Thus, the extent to which the observed functional differences relates to anatomy is unknown and requires investigation.

Moreover, the challenges in the reproducibility of neuroimaging findings (Griffanti et al., 2016) and the influence of small sample size in the reliability of results (Button et al., 2013) have been much discussed in the current scientific literature. A major strength of this study is the reproducibility of most of the results in a large sample of similar individuals from the ABIDE II dataset (Di Martino et al., 2017). On the other hand, there are limitations that need to be taken into consideration. The estimation of cerebellar zones coupled to cerebral regions was compromised by the fact that there was no full coverage of the cerebellum in most individuals, 
particularly in the posterior region. Therefore, further studies are required to comprehensively examine ASD-related differences in cortico-cerebellar functional connectivity. Importantly, both the ABIDE I and ABIDE II datasets are multicentric, with heterogeneous acquisition parameters across sites. Thus, to minimize the site effect in our analysis, we included it as a covariate in group-level statistics as described in the section 2.3. It is also important to highlight that since the ROIs were divided according to anatomical regions, they could exhibit functional heterogeneity, possibly leading to ambiguous functional connectivity.

In conclusion, our results suggest that ASD displays atypical reduced intrinsic functional cortico-cerebellar connectivity in specific networks, which is consistent with the idea that ASD is a disorder characterized by abnormalities in neural connections (Hoppenbrouwers et al., 2014).

\section{AUTHOR CONTRIBUTIONS}

TR and AF conceived the analyses. TR conducted the analyses. $\mathrm{TR}, \mathrm{JB}, \mathrm{JS}$, and AF analyzed the results and reviewed the

\section{REFERENCES}

Alexander, A. L., Lee, J. E., Lazar, M., Boudos, R., DuBray, M. B., Oakes, T. R., et al. (2007). Diffusion tensor imaging of the corpus callosum in autism. Neuroimage 34, 61-73. doi: 10.1016/j.neuroimage.2006.08.032

American Psychiatric Association (2013). Diagnostic and Statistical Manual of Mental Disorders (DSM-5®). Arlington, VA: American Psychiatric Pub.

Barnea-Goraly, N., Kwon, H., Menon, V., Eliez, S., Lotspeich, L., and Reiss, A. L. (2004). White matter structure in autism: preliminary evidence from diffusion tensor imaging. Biol. Psychiatry 55, 323-326. doi: 10.1016/j.biopsych.2003.10.022

Bauman, M. L., and Kemper, T. L. (2005). Neuroanatomic observations of the brain in autism: a review and future directions. Int. J. Dev. Neurosci. 23, 183-187. doi: 10.1016/j.ijdevneu.2004.09.006

Baumann, O., Borra, R. J., Bower, J. M., Cullen, K. E., Habas, C., Ivry, R. B., et al. (2015). Consensus paper: the role of the cerebellum in perceptual processes. Cerebellum 14, 197-220. doi: 10.1007/s12311-014-0627-7

Ben Bashat, D., Kronfeld-Duenias, V., Zachor, D. A., Ekstein, P. M., Hendler, T., Tarrasch, R., et al. (2007). Accelerated maturation of white matter in young children with autism: a high b value DWI study. Neuroimage 37, 40-47. doi: 10.1016/j.neuroimage.2007.04.060

Benjamini, Y., and Hochberg, Y. (1995). Controlling the false discovery rate: a practical and powerful approach to multiple testing. J. R. Stat. Soc. Ser. B Methodol. 57, 289-300. doi: 10.1111/j.2517-6161.1995.tb02031.x

Biswal, B., Yetkin, F. Z., Haughton, V. M., and Hyde, J. S. (1995). Functional connectivity in the motor cortex of resting human brain using echoplanar MRI. Magn. Reson. Med. 34, 537-541. doi: 10.1002/mrm.19103 40409

Brito, A. R., Vasconcelos, M. M., Domingues, R. C., Hygino da Cruz, L. C., Rodrigues Lde, d. S., Gasparetto, E. L., et al. (2009). Diffusion tensor imaging findings in school-aged autistic children. J. Neuroimaging 19, 337-343. doi: 10.1111/j.1552-6569.2009.00366.x

Buckner, R. L. (2013). The cerebellum and cognitive function: 25 years of insight from anatomy and neuroimaging. Neuron 80, 807-815. doi: 10.1016/j.neuron.2013.10.044

Buckner, R. L., Krienen, F. M., Castellanos, A., Diaz, J. C., and Yeo, B. T. T. (2011). The organization of the human cerebellum estimated by intrinsic functional connectivity. J. Neurophysiol. 106, 2322-2345. doi: 10.1152/jn. 00339.2011 manuscript. All authors read and approved the final version of the manuscript.

\section{ACKNOWLEDGMENTS}

TR was partially supported by the Coordenação de Aperfeiçoamento de Pessoal de Nível Superior - Brasil (CAPES) (051/2013) and CNPq (141492/2017-1). AF was partially supported by the São Paulo Research Foundation (2013/07375-0, 2015/01587-0, 2016/13422-9, 2018/17996-5), CNPq (304876/2016-0), CAPES (Finance Code 001), Alexander von Humboldt Foundation, and Medical Academy of SciencesNewton Advanced Fund. The authors would like to thank the ABIDE Consortium for making the fMRI database publicly available.

\section{SUPPLEMENTARY MATERIAL}

The Supplementary Material for this article can be found online at: https://www.frontiersin.org/articles/10.3389/fnsys. 2018.00074/full\#supplementary-material
Button, K. S., Ioannidis, J. P., Mokrysz, C., Nosek, B. A., Flint, J., Robinson, E. S., et al. (2013). Power failure: why small sample size undermines the reliability of neuroscience. Nat. Rev. Neurosci. 14:365. doi: 10.1038/nrn3475

Catani, M., Jones, D. K., Daly, E., Embiricos, N., Deeley, Q., Pugliese, L., et al. (2008). Altered cerebellar feedback projections in Asperger syndrome. Neuroimage 41, 1184-1191. doi: 10.1016/j.neuroimage.2008.03.041

Christensen, D. L. (2016). Prevalence and characteristics of autism spectrum disorder among children aged 8 years - autism and developmental disabilities monitoring network, 11 sites, United States, 2012. MMWR Surveill. Summar. 65,1-23. doi: 10.15585/mmwr.ss6503al

Courchesne, E., Yeung-Courchesne, R., Press, G. A., Hesselink, J., and Jernigan, T. (1988). Hypoplasia of cerebellar vermal lobules VI and VII in autism. New Engl. J. Med. 318, 1349-1354. doi: 10.1056/NEJM198805263182102

Crippa, A., Del Vecchio, G., Busti Ceccarelli, S., Nobile, M., Arrigoni, F., and Brambilla, P. (2016). Cortico-cerebellar connectivity in autism spectrum disorder: what do we know so far? Front. Psychiatry 7:20. doi: 10.3389/fpsyt.2016.00020

Di Martino, A., O'Connor, D., Chen, B., Alaerts, K., Anderson, J. S., Assaf, M., et al. (2017). Enhancing studies of the connectome in autism using the autism brain imaging data exchange II. Sci. Data 4:170010. doi: 10.1038/sdata.2017.10

Ecker, C., Bookheimer, S. Y., and Murphy, D. G. M. (2015). Neuroimaging in autism spectrum disorder: brain structure and function across the lifespan. Lancet Neurol. 14, 1121-1134. doi: 10.1016/S1474-4422(15)00050-2

Elsabbagh, M., Divan, G., Koh, Y.-J., Kim, Y. S., Kauchali, S., Marcín, C., et al. (2012). Global prevalence of autism and other pervasive developmental disorders. Autism Res. 5, 160-179. doi: 10.1002/aur.239

Fatemi, S. H., Aldinger, K. A., Ashwood, P., Bauman, M. L., Blaha, C. D., Blatt, G. J., et al. (2012). Consensus paper: pathological role of the cerebellum in autism. Cerebellum 11, 777-807. doi: 10.1007/s12311-012-0355-9

Fatemi, S. H., Halt, A. R., Realmuto, G., Earle, J., Kist, D. A., Thuras, P., et al. (2002). Purkinje cell size is reduced in cerebellum of patients with autism. Cell. Mol. Neurobiol. 22, 171-175. doi: 10.1023/A:1019861721160

Floris, D. L., Barber, A. D., Nebel, M. B., Martinelli, M., Lai, M.-C., Crocetti, D., et al. (2016). Atypical lateralization of motor circuit functional connectivity in children with autism is associated with motor deficits. Mol. Autism 7:35. doi: 10.1186/s13229-016-0096-6

Fox, M. D., and Raichle, M. E. (2007). Spontaneous fluctuations in brain activity observed with functional magnetic resonance imaging. Nat. Rev. Neurosci. 8, 700-711. doi: 10.1038/nrn2201 
Glickstein, M. (1992). The cerebellum and motor learning. Curr. Opin. Neurobiol. 2, 802-806.

Griffanti, L., Rolinski, M., Szewczyk-Krolikowski, K., Menke, R. A., Filippini, N., Zamboni, G., et al. (2016). Challenges in the reproducibility of clinical studies with resting state fmri: an example in early parkinson's disease. Neuroimage 124, 704-713. doi: 10.1016/j.neuroimage.2015.09.021

Hanaie, R., Mohri, I., Kagitani-Shimono, K., Tachibana, M., Azuma, J., Matsuzaki, J., et al. (2013). Altered microstructural connectivity of the superior cerebellar peduncle is related to motor dysfunction in children with autistic spectrum disorders. Cerebellum 12, 645-656. doi: 10.1007/s12311-013-0475-x

Hashimoto, T., Tayama, M., Murakawa, K., Yoshimoto, T., Miyazaki, M., Harada, M., et al. (1995). Development of the brainstem and cerebellum in autistic patients. J. Autism Dev. Dis. 25, 1-18.

Hoche, F., Guell, X., Sherman, J. C., Vangel, M. G., and Schmahmann, J. D. (2016). Cerebellar contribution to social cognition. Cerebellum 15, 732-743. doi: 10.1007/s12311-015-0746-9

Hoppenbrouwers, M., Vandermosten, M., and Boets, B. (2014). Autism as a disconnection syndrome: a qualitative and quantitative review of diffusion tensor imaging studies. Res. Autism Spect. Disord. 8, 387-412. doi: 10.1016/j.rasd.2013.12.018

Just, M. A., Keller, T. A., Malave, V. L., Kana, R. K., and Varma, S. (2012). Autism as a neural systems disorder: a theory of frontal-posterior underconnectivity. Neurosci. Biobehav. Rev. 36, 1292-1313. doi: 10.1016/j.neubiorev.2012.02.007

Keren-Happuch H., Chen, S.-H. A., Ho, M.-H. R., and Desmond, J. E. (2014). A meta-analysis of cerebellar contributions to higher cognition from pet and fMRI studies. Hum. Brain Mapp. 35, 593-615. doi: 10.1002/hbm.22194

Khan, A. J., Nair, A., Keown, C. L., Datko, M. C., Lincoln, A. J., and Müller, R.A. (2015). Cerebro-cerebellar resting-state functional connectivity in children and adolescents with autism spectrum disorder. Biol. Psychiatry 78, 625-634. doi: 10.1016/j.biopsych.2015.03.024

Lee, J. E., Bigler, E. D., Alexander, A. L., Lazar, M., DuBray, M. B., Chung, M. K., et al. (2007). Diffusion tensor imaging of white matter in the superior temporal gyrus and temporal stem in autism. Neurosci. Lett. 424, 127-132. doi: 10.1016/j.neulet.2007.07.042

Libero, L. E., Burge, W. K., Deshpande, H. D., Pestilli, F., and Kana, R. K. (2016). White matter diffusion of major fiber tracts implicated in autism spectrum disorder. Brain Connect. 6, 691-699. doi: 10.1089/brain.2016.0442

Limperopoulos, C., Chilingaryan, G., Sullivan, N., Guizard, N., Robertson, R. L., and du Plessis, A. J. (2014). Injury to the Premature cerebellum: outcome is related to remote cortical development. Cereb. Cortex 24, 728-736. doi: 10.1093/cercor/bhs354

Maximo, J. O., Cadena, E. J., and Kana, R. K. (2014). The implications of brain connectivity in the neuropsychology of autism. Neuropsychol. Rev. 24, 16-31. doi: 10.1007/s11065-014-9250-0

Mostofsky, S. H., Powell, S. K., Simmonds, D. J., Goldberg, M. C., Caffo, B., and Pekar, J. J. (2009). Decreased connectivity and cerebellar activity in autism during motor task performance. Brain 132(Pt 9), 2413-2425. doi: 10.1093/brain/awp088

Müller, R.-A., Shih, P., Keehn, B., Deyoe, J. R., Leyden, K. M., and Shukla, D. K. (2011). Underconnected, but how? A survey of functional connectivity MRI studies in autism spectrum disorders. Cereb. Cortex 21, 2233-2243. doi: 10.1093/cercor/bhq296

Nair, A., Treiber, J. M., Shukla, D. K., Shih, P., and Müller, R.-A. (2013). Impaired thalamocortical connectivity in autism spectrum disorder: a study of functional and anatomical connectivity. Brain 136, 1942-1955. doi: 10.1093/brain/ awt079

Noroozian, M. (2014). The role of the cerebellum in cognition: beyond coordination in the central nervous system. Neurol. Clin. 32, 1081-1104. doi: 10.1016/j.ncl.2014.07.005
Ogawa, S., Lee, T. M., Kay, A. R., and Tank, D. W. (1990). Brain magnetic resonance imaging with contrast dependent on blood oxygenation. Proc. Natl. Acad. Sci. U.S.A. 87, 9868-9872.

O’Reilly, J. X., Beckmann, C. F., Tomassini, V., Ramnani, N., and JohansenBerg, H. (2010). Distinct and overlapping functional zones in the cerebellum defined by resting state functional connectivity. Cereb. Cortex 20, 953-965. doi: 10.1093/cercor/bhp157

Power, J. D., Barnes, K. A., Snyder, A. Z., Schlaggar, B. L., and Petersen, S. E. (2012). Spurious but systematic correlations in functional connectivity MRI networks arise from subject motion. Neuroimage 59, 2142-2154. doi: 10.1016/j.neuroimage.2011.10.018

Ramnani, N. (2006). The primate cortico-cerebellar system: anatomy and function. Nat. Rev. Neurosci. 7, 511-522. doi: 10.1038/nrn1953

Schmahmann, J. D. (2004). Disorders of the cerebellum: ataxia, dysmetria of thought, and the cerebellar cognitive affective syndrome. J. Neuropsychiatry Clin. Neurosci. 16, 367-378. doi: 10.1176/jnp.16.3.367

Schmahmann, J. D. (2010). The role of the cerebellum in cognition and emotion: personal reflections since 1982 on the dysmetria of thought hypothesis, and its historical evolution from theory to therapy. Neuropsychol. Rev. 20, 236-260. doi: 10.1007/s11065-010-9142-x

Shukla, D. K., Keehn, B.,and Müller, R. A. (2010). Tract-specific analyses of diffusion tensor imaging show widespread white matter compromise in autism spectrum disorder. J. Child Psychol. Psychiatry 52, 286-295. doi: 10.1111/j.1469-7610.2010.02342.x

Smith, S. M., Fox, P. T., Miller, K. L., Glahn, D. C., Fox, P. M., Mackay, C. E., et al. (2009). Correspondence of the brain's functional architecture during activation and rest. Proc. Natl. Acad. Sci. U.S.A. 106, 13040-13045. doi: 10.1073/pnas.0905267106

Stoodley, C. J., and Schmahmann, J. D. (2009). Functional topography in the human cerebellum: a meta-analysis of neuroimaging studies. Neuroimage 44 , 489-501. doi: 10.1016/j.neuroimage.2008.08.039

Tzourio-Mazoyer, N., Landeau, B., Papathanassiou, D., Crivello, F., Etard, O., Delcroix, N., et al. (2002). Automated anatomical labeling of activations in SPM using a macroscopic anatomical parcellation of the MNI MRI single-subject brain. Neuroimage 15, 273-289. doi: 10.1006/nimg.2001.0978

Verly, M., Verhoeven, J., Zink, I., Mantini, D., Peeters, R., Deprez, S., et al. (2014). Altered functional connectivity of the language network in ASD: role of classical language areas and cerebellum. Neuroimage Clin. 4, 374-382. doi: 10.1016/j.nicl.2014.01.008

Webb, S. J., Sparks, B.-F., Friedman, S. D., Shaw, D. W. W., Giedd, J., Dawson, G., et al. (2009). Cerebellar vermal volumes and behavioral correlates in children with autism spectrum disorder. Psychiatry Res. Neuroimaging 172, 61-67. doi: 10.1016/j.pscychresns.2008.06.001

Wolff, J. J., Gu, H., Gerig, G., Elison, J. T., Styner, M., Gouttard, S., et al. (2012). Differences in white matter fiber tract development present from 6 to 24 months in infants with autism. Am. J. Psychiatry 169, 589-600. doi: 10.1176/appi.ajp.2011.11091447

Conflict of Interest Statement: The authors declare that the research was conducted in the absence of any commercial or financial relationships that could be construed as a potential conflict of interest.

Copyright (๐ 2019 Ramos, Balardin, Sato and Fujita. This is an open-access article distributed under the terms of the Creative Commons Attribution License (CC BY). The use, distribution or reproduction in other forums is permitted, provided the original author(s) and the copyright owner(s) are credited and that the original publication in this journal is cited, in accordance with accepted academic practice. No use, distribution or reproduction is permitted which does not comply with these terms. 